\title{
European Council's Recent Revision in MedDev 2.7.1 Guidelines (Revision 4) - Quantifying its Change and its Impact
}

\section{Ashish ${ }^{*}$}

Domain Consultant - Medical Devices, Tata Consultancy Services, Mumbai, India

"Corresponding author: Ashish I, Domain Consultant - Medical Devices, Tata Consultancy Services, Second floor, Tiffany, Hirannadani Estate Thane (W), 401107, Mumbai, India, Tel: +919833316130; E-mail: ashish.indani@tcs.com

Received date: 30, March 2017; Accepted date: 18, April 2017; Published date: 25, April 2017

Copyright: $\odot 2017$ Ashish I. This is an open-access article distributed under the terms of the Creative Commons Attribution License, which permits unrestricted use, distribution and reproduction in any medium, provided the original author and source are credited.

\begin{abstract}
Latest updates in guidelines of European Union for medical device clinical evaluation report (CER) is particularly in line with the shift of focus of evaluation process from "verification of safety and performance" to justification of "sufficient evidence conforming to the essential requirements" and subsequent change in the approach of clinical evaluation from a subjective justification based upon collection of information to an objective, holistic and logical clinical justification based upon clear and rational methods. Stating that the clinical evaluators need to have an experience of about 10 years in clinical research and medical writing, requiring an all-inclusive state-of-the-art for device-treated disease, and specifying clinical data sources, the guidelines have given a direction for CER to be logical and objective. The requirement to align manufacturer supplied information such as instructions for use, Clinical Investigations Report and CER is indicative of the holistic approach.
\end{abstract}

These guidelines are not effective immediately, however, their merits and value addition as well as its immediate implantation is practical and operationally beneficial for the manufacturers. They have removed ambiguities about the qualifications, acceptability standards of performance and safety of the device, scope of clinical evaluation etc. and have set expectations.

The guidelines are supposed to bring about two major changes in the CER trends. The first change will be in the trend of personnel. We can now expect increased engagement for medical writer with 10 years of more experience. In addition, organizations with personnel having diverse background will be in a better position to deliver the CER. Due to increased engagement in PMS, PMCF and registry activities, increased literature search and summarization requirements and frequent update of $C E R$, need for automation will increase.

Keywords: MedDEV; Clinical evaluation; CER; Medical device clinical evaluation; Medical device substantial equivalence

\section{Introduction}

Revision 4 of the MEDDEV 2.7.1 guidelines are released in the month of June 2016 [1]. However, there is no clear mention about the effective date. Therefore, some notified bodies may continue to accept the CER still based upon the older version. However, for many reasons, starting to use the latest revision 4 is recommended. As the guidelines are already published, the regulatory bodies may make compliance with these guidelines a requirement in the near future. Instead of rushing when compliance with revision 4 is mandatory, quick implementation will provide a better resource management opportunity. The current revision is more viable operationally and practically. Revised instructions included in this revision enable manufacturers to conduct an effective clinical evaluation and demonstration of the scientific validity of data and conclusions.

In this revision, we can observe a shift of focus in the evaluation process from "verification of safety and performance" to justification of "sufficient evidence conforming to the essential requirements" and subsequent change in the approach of clinical evaluation from a subjective justification based upon collection of information to an objective, holistic and logical clinical justification based upon clear and rational methods. Even if in both cases, the main purpose is to justify that the device meets the intended clinical purpose, in the current revision, it mentions that CER should be the sufficient clinical evidence to justify essential requirements as per the European Commission (EC) directives. For effective use of the CER for this purpose, three major documents which pertain to essential requirement justification need to be aligned with each other. These documents are:

Information materials supplied by the manufacturer (the labelling, instructions for use, available promotional materials, including accompanying documents foreseen by the manufacturer).

The clinical evaluation report (the device description, claims, intended use and anticipated events used for the clinical evaluation, other contents of the clinical evaluation report).

The available clinical data (such as results of clinical investigations, publications, PMS studies, etc.).

With this alignment, at an operational level, this document will be instrumental in delivering high quality clinical evaluation as per requirements of recent medical device reporting (MDR) changes and requirements for post-market surveillance (PMS) and post-market clinical follow-up (PMCF). The PMS and PMCF requirement is repronounced in these documents as well.

From revision 3 to revision 4, certain definitions and acceptable methodology is used to analyze data that have undergone certain changes to align with the amendment of expectations set by change in 
the approach. They now include more prescriptive expectations. With a new version of ISO 14155 released in 2011, the definitions and methods are updated. In addition, now the requirements are more aligned with the European Commission (EC) requirements than with the GHTF as in version 3. In revision 4, there is a complete section (section 6) on the contents of CER, rather than only a little information in section 1: 'Introduction' of revision 3 [2]. This description is consolidated with more clarity and details. The revision 4 provides clarity on confirmation of conformity to essential requirements and comprehensible information on purpose of clinical evaluation is mentioned. The scope of CER in made extremely clear under section 7 in revision 4, whereas, revision 3 mentions scope extent of the guidance document only. Revision 4 defined acceptance levels of the guidance document [1-4].

In addition, there are a few specific changes from the previous versions, in various sections. These changes are discussed by section further in this document. This revision has addressed several ambiguities and subjectivities left behind by previous versions. In a nutshell, the new version is more precise in answering the $\mathrm{W} 4 \mathrm{H}$ questions. Before we answer the W4H questions, looking into the definition changes in the introduction section will build the basic information and background.

\section{Changes in the Definitions}

In line with changes in justification approach, various additions and updates in the definitions are observed in revision 4. Certain changes are further influenced by updates in the MDR requirements in 2016 and updates in ISO 14155 in 2011. The definition of adverse event is amended to be in line with the ISO 14155-2011 definition [5-7]. The definition in revision 3 was limited only to the device under evaluation and the subject in whom the device is used, whether device is related or not. The definition of clinical data and clinical evaluation are amended with the reference change from GHTH SG5 N2R8 to the MDD and AIMDD [8-11]. In revision 4, the sources of the clinical data are clearly specified. The most important point to consider is the change of definition of clinical evaluation, which brings about a major difference. The major component changes and its effect are summarized in Table 1.

\begin{tabular}{|c|c|c|}
\hline & Revision 3 & Revision 4 \\
\hline Complete definition & $\begin{array}{l}\text { The assessment and analysis of clinical } \\
\text { data pertaining to a medical device to } \\
\text { verify the clinical safety and performance } \\
\text { of the device when used as intended by } \\
\text { the manufacturer }\end{array}$ & $\begin{array}{l}\text { A methodologically sound ongoing procedure to collect, appraise and analyze clinical data } \\
\text { pertaining to a medical device and to evaluate whether there is sufficient clinical evidence to } \\
\text { confirm compliance with relevant essential requirements for safety and performance when } \\
\text { using the device according to the manufacturer's instructions for use Note: In exceptional } \\
\text { cases where an instruction for use is not required, the collection, analysis and assessment } \\
\text { are conducted taking into account the generally recognized modalities of use }\end{array}$ \\
\hline Process consideration & $\begin{array}{l}\text { Assessment and analysis of clinical data } \\
\text { pertaining to the device }\end{array}$ & Methodologically sound ongoing procedure to collect, appraise and analyze clinical data \\
\hline Focus & $\begin{array}{l}\text { To verify the clinical safety and } \\
\text { performance of the device }\end{array}$ & $\begin{array}{l}\text { To evaluate whether there is sufficient clinical evidence to confirm compliance with relevant } \\
\text { essential requirements for safety and performance }\end{array}$ \\
\hline Basis of evaluation & $\begin{array}{l}\text { When used as intended by the } \\
\text { manufacturer }\end{array}$ & When using the device according to the manufacturer's instructions for use \\
\hline Outcome & Compliance with intended use & Clinical performance \\
\hline
\end{tabular}

Table 1: Highlights of changes of definitions between revision 3 and revision 4 of MedDEV2.7.1 guidelines.

In Revision 4, a few new definitions are added. These terms are fundamentally related with the clinical research, which strengthens the concept that the CER process requires a transformation of approach. This new set of terms is in line with the overall strategy of this revision of making clinical evaluation a holistic and logical document justifying clinical safety and performance. The definitions added are bias, device registry, clinical use, equivalent device, feasibility study, hazard, hazard due to substances and technologies, incident, information materials supplied by the manufacturer, intended purpose, PMCF plan, PMCF study, risk, risk management, sufficient clinical evidence. The last term in the list is a major eye-catcher.

As discussed, the revision 4 has better answers to $\mathrm{W} 4 \mathrm{H}$ questions in process perfection. The W4H questions are What, Why, When, Who and How. Here are MedDev 2.7.1 revision 4 answers to these questions [1]:

What are the contents of CER? Link between CER, post-market surveillance and post-market clinical follow-up.

There are no major changes in the content requirements. In general, the principles of clinical evaluation process remain the same. The manufacturers can continue using clinical data based on pre and post marketing investigations, complaints, unpublished reports, feedback or experience letters from the investigators and data from other devices to justify compliance with the relevant essential requirements. However, there is a specific addition. The clinical evaluation report now reinforces the links between clinical evaluation, post-market surveillance (PMS) and post-market clinical follow-up (PMCF). In CER based upon previous version of the guidance, due to inadequate control on the contents, the information on PMCF, and patient consequences in PMS or materiovigilance as not included in the CER. The updates coming from such studies/reports was not incorporated in the updates of CER. In addition, due to gross under-reporting of clinical consequences of the device in patients, the information on PMS was not scientifically valid. Therefore, in the current version of CER, the information from all PMCF and patient consequences of the PMS/PMS follow-up studies need to be updated to CER as soon as available. For this, the CER needs to be updated. The data from clinical investigation, whether published or not, must be included in the CER. In accordance with the objectives and scope of CER in section 7, the 
content of clinical evaluation report must be adequate to establish safety, performance and justification of essential requirements.

\section{Role of Notified Bodies}

As per appendix 12, the notified bodies will now require planned PMCF and their conclusions documented within the CER. In most cases earlier, post marketing surveillance and complaints management data was included in the clinical evaluation, where gross underreporting was observed. Therefore, now the notified bodies will be responsible to check that the plan for PMCF is adequate and is aligned/designed to mitigate identified gaps. The notified bodies will also be responsible for assessment of manufacturers' CER, PMS and PMCF procedures.

\section{Why clinical evaluation? Section 7 - clarifying CER objectives}

Besides several carry-forwards from version 3, the stage " 0 " of clinical evaluation process in the guidelines (section 7) has mentioned clearly about setting correct objectives and scopes for the clinical evaluation. Unlike the earlier versions, this version requires that CER objectives or scopes be more precise and clearly linked to:

Specific safety, performance, and risk/benefit endpoints of the device.

The performance claims made by the manufacturer.

This change will lead the process to be scientifically driven. The major purpose of defined scope and objectives is to eliminate bias towards the end when the data is evaluated.

\section{Why clinical evaluation? Section 8.2: Establishing current trends or state-of-the-art and justifying where the device fits}

Section 8.2 reveals the major reason for setting up medical/clinical research qualifications of the authors of clinical evaluation report. Differing from the earlier guidance on clinical aspect, the updated guidance requires the manufacturer to identify a state-of-the-art (standard of care) for a particular disease condition or treatment/ therapy in which the device is used. State-of-the-art or the standard of care can be established from the data from predicates, safety/ performance data from other available treatments etc. and information collected can establish clinical safety and performance endpoints.

This state-of-the-art will be included in CER and referred throughout for comparison with the device under evaluation and is specified in the section 8.2 of the guidance. The principle idea of comparison is to set a reference standard for clinically acceptable v/s. avoidable risks and residual risks. As a result of comparison, the devices can be classified into two buckets. The devices which do not meet the clinically acceptable risks, however, can be justified on basis of efficacy and preventable risks. The second bucket of devices contains the device with comparable risk profile to the established standards. Unless the claims of the device pertain to superior safety or efficacy, the devices in this bucket do not need any additional justification. To claim its device is superior in safety or efficacy or both, correct comparators will be required. Superiority cannot be claimed by comparing performance of a device to devices which historically exhibited poor performance.

\section{Why clinical evaluation? Clarifying scientific validity of the data}

The updated guidance clarifies the necessity to obtain sufficient clinical evidence (amount and quality) in order to guarantee the scientific validity of the conclusions. It also requires justification of claims for its scientific validity and justification. The guidance details the process to evaluate methodical quality and scientific validity, and maps out each stage of the clinical evaluation process to ensure completeness and objectivity. This section of the guidance also elucidates literature search, retrieval methods, data analysis, and demonstration of conformity.

Evaluators need to examine the methods used to generate data and then evaluate the extent to which the performance or safety outcomes can be attributed to intervention with the device. Relevant factors in the evaluation include random errors, bias, inadequate disclosure of information, misinterpretation, or other discombobulate factors. Overall, though, the clinical evaluation process remains much the same.

\section{Why clinical evaluation? Equivalence to data for equivalent devices}

This section has been expanded from the previous versions and the requirements are described in Appendix 1. Equivalence still is assessed with respect to clinical, technological, and biological parameters, but the revised guidance requires more documented information for each device in order to demonstrate equivalence to those parameters.

Evaluators may wish to compare several equivalent devices, and in such cases, each device compared to the device under evaluation should be fully investigated, demonstrated, and described in the CER. Populations should be equivalent to the European Union, as well, so manufacturers need to determine if the existing data is relevant; it could be a struggle to justify equivalence if there are significant differences in patient size, weight, etc., in data gleaned from populations outside of the EU.

A significant level of detail must be provided to demonstrate equivalence; the guidance indicates that the differences between the device under evaluation and equivalent devices be identified, fully disclosed, and evaluated. It should be clearly explained how any differences do not affect the performance or clinical safety of the device under evaluation. This process includes analysis of design differences, specifications, comparative drawings and diagrams, descriptions of differences in physical and chemical properties, material characterization and comparative testing in accordance with ISO 10993, and a look at the impact of the devices' manufacturing processes. All of this information should be summarized in the CER to support information in the technical file, and the technical file should refer to the location of all the supporting documentation.

Furthermore, equivalent devices must be CE marked and used in accordance with their intended purpose, as documented in instructions for use (IFU). Exceptions can be considered when the equivalent device is not CE marked, but a thorough justification with a gap analysis must be included in the CER.

Finally, a contract must be in place with equivalent device manufacturers, allowing full access to their data on an on-going basis. This is particularly challenging for manufacturers, as such information might be confidential, sensitive, or unavailable to the manufacturer. Notified bodies are encouraged in the guidance to ensure that 
manufacturers make every attempt to obtain this information and, if collecting the said information is not feasible, manufacturers should at least provide physical, mechanical, and chemical characterization requirements through comparative testing.

\section{Why clinical evaluation? Clarifying risk/benefit and justifying claims}

A quantification of the benefit and risk, as well as an evaluation of the overall risk/benefit profile, is required under Appendix 7.2. Measurable benefits for the patient include quantifiable improvements in the clinical outcome, the average duration of these benefits and the probability of the patient experiencing one of these benefits. These benefits should be weighed against a similar evaluation of the device's clinical risks, including the probability of a harmful event; the severity, number and rates of harmful events; and the duration of a harmful event. The ISO 14971 Standard is a worthy tool for manufacturers developing a risk/benefit plan.

\section{When to perform clinical evaluation or update CER?}

Earlier version had a detailed description of when to undertake a clinical evaluation report. However, there was a limited clarity about the frequency of clinical evaluation report in earlier revisions. In this revision, the European Commission (EC) has included details of requirements for the frequency of evaluation report updates. The clinical evaluation is an on-going process; hence a clinical evaluation report must be updated as:

The first clinical evaluation report is published for CE marking.

Revising CER at justified and defied regular intervals.

\section{NB 1}

For very low risk (GHTF class A, EU class I), that are established, updates every two to five years would be a rational upgradation period, unless they have undergone some changes.

For novel devices or devices that impose significant risks pertaining to design or intended use (such as use in high-risk patient populations or into the critical disease condition) the CER updates must be updated atleast annually.

For Class II(a) device manufacturers must update the report atleast every two years.

For Class II(b) and Class III devices and implantable devices, manufacturers must update the report atleast annually. In addition, considering MDR requirements, for class III device manufacturers must submit the report for review by their notified body, through EUDAMED.

When new PMS/PMCF data is available especially if the information could affect the conclusion of the clinical evaluation report (CER).

\section{NB 2}

If the PMS data received has the potential to change the current evaluation, manufacturers must assess the need to update the report.

The PMS activities should also be considered in line with the medical device reporting requirements. Accordingly, the periodic safety update report (PSUR) and the summary of clinical performance for classes II(a), II(b) and III, PSUR must be maintained throughout the lifetime of the device.

CER and PSUR should address conclusions of the post-market surveillance data and associated corrective and preventive actions (CAPAs) or field corrective actions (FCA).

\section{When is clinical investigation required? - Appendix -2}

Clinical evaluation is a continuous process and general requirement is sufficient clinical evidence to demonstrate compliance of all essential requirements. Clinical investigation is required in a few cases to justify such compliance. Appendix 2 of the current guidelines have laid down the considerations on how to assess whether clinical investigation is needed.

Clinical considerations: All indications and conditions of intended use, all target patient populations, all variants of the device under investigation including its various models and sizes.

Scientific sanctity of the data: The data must be scientifically sound and should demonstrate compliance with state-of-the-art as established under section 8.2, discussed later in this document.

Insufficient data to support all facts: In clinical evaluation, a Gap analysis is undertaken from state-of-the-art. If certain gaps could not be established from other sources, then a clinical investigation is required.

Type of device: As per Council directives as well as other guidance documents, all implantable, high-risk, and Class III devices always require clinical investigation. However, if a clinical evaluation suffices to justify with strong, risk-based clinical evidence, clinical investigation may be escaped.

Tenure of technology: The devices with established use or based upon a proven and time-tested technology does not necessarily require a clinical investigation. Devices with some minor changes in the clinical application of the established technology can be justified through clinical evaluation report. However, devices with new or unproven technologies require a clinical investigation as they will be considered as "new clinical use". The new clinical use will include:

Completely new technology e.g. Bioreabsorbable Vascular Scaffold.

A new device with partially new technology, materials or design features in an existing indication for the basic technology e.g.: Drug eluting stent with new drug without polymer.

Extension of an established technology to a new indication or intended purpose e.g. drug eluting stent in carotid or below the knee use.

New claims pertaining to target populations, risks recognized and types of users.

In addition, use of active or medicinal substances, use of animal tissues, and extent of invasiveness of the device is an important aspect in determination of the need for clinical investigation. If in consideration of manufacturer, the clinical evaluation is enough to justify compliance with essential requirements and state-of-the-art, a paragraph in this pursuit needs to be included in the CER.

Who should write clinical evaluation report? - Report Author and Evaluator Qualifications.

Before revision 4 provided details, there was quite significant ambiguity and subsequent differences of the opinions about 
qualifications of the CER author's qualifications and experience. The section 6.4 provides clarification regarding required qualifications of the CER process team members. The guidelines mentions "suitably qualified individual or a team" It further clarifies the qualifications and experiences as: Knowledge.

Research methodology (including clinical investigation design and biostatistics).

Information management (e.g. scientific background or librarianship qualification; experience with relevant databases such as Embase and Medline).

Regulatory requirements; and medical writing.

The device technology and its application.

Diagnosis and management of the conditions intended to be diagnosed or managed by the device, knowledge of other medical options, treatment standards and technology (e.g. specialist clinical expertise in the relevant medical specialty).

\section{Qualifications and experience:}

Post-graduate experience in a relevant science or in medicine

Training and experience in medical writing, systematic review and clinical data appraisal

Degree plus five years of relevant professional experience OR

If degree is not a mandatory requirement for the given task, 10 years of relevant experience

NB: Not all members need to have these qualifications and experience, but the skill sets should somehow be represented within the team.

Changes in skill set for notified bodies: The notified bodies will need to assess the data presented in the CER, the validity of the conclusions drawn by the manufacturer, and the conformity of the device to relevant essential requirements. Therefore, now they will need to restructure the review teams to include individuals with relevant clinical experience, as well, such as doctors, nurses, clinicians, etc.

How? Process and content of the clinical evaluation report.

Most of the contents of CER continues to have basic information related to the device, unique device identification (UDI) information, intended purpose, harmonized standards, training for users, residual risks, indications, descriptions of differences from previous models, and summaries of the clinical evaluation report and post-market clinical follow-up etc. Additionally, this revision of the guideline includes guidance on some non-technical and non-clinical data that would surrogate the clinical information. This guidance is in line with MDR requirements under section 60c (PSUR) and 26 (Safety and Clinical Performance). It states that sales data should be included, along with an estimate of the population exposed to the device and possibly a usage frequency.

The revised guidelines have set expectations towards clinical evaluation and sufficient clinical data. The guidelines require setting state-of-the-art for medical condition for which the device is intended to be used. This state-of-the-art can be driven from various options of the treatments and their residual risks. From literature search and complaints data of the device, the clinical evaluator can establish the state-of-the-art and relation of the device with such state-of-the-art. This clause is the most important change in the process of clinical evaluation from earlier version. Due to lack of a set standard, the justification of device safety and performance was extremely subjective and was influenced by knowledge and bias factor of the clinical evaluator [12].

The clinical evaluation process is explained in 5 steps, from stage 0 to stage 4. (NB: there were three stages in clinical evaluation process as per revision 3.) Section 7 has information about writing the scope of clinical evaluation report and also provides clear information on tasks included in initial CER and its updates. The new guidelines also require scopes to include justifying and substantiating the marketing claims of the device. The content of this section remains the same. Certain important carry forwards from the previous version are that the manufacturers need to start with the device specification, identify related essential requirements to support clinical evidence, define the scope of the clinical evaluation and, ultimately, define specific and measurable objectives. However, unlike the previous guidelines, this version has scope for equivalence at clinical, technical and biological plains. The stage 0 facilitates capture of technical and biological information in the CER. The typical controls of a device are mentioned in appendix 3 of the guidelines. It is taken from the guidelines to explain regulators' consideration of the process map.

\section{How? Literature search}

The source of literature is clarified in Appendix 4 as it was in the earlier guidelines, but with a better clarity. Appendix A5 defines key elements of the literature search and review protocol. The literature search protocol now will need to be set for objective, unbiased, and systematic outputs of the research articles. The search phrases for literature review need to be specific, and should define three key elements:

\section{Population/disease for whom the device is intended}

Intervention and controls: Outcomes (mortality, qualify of life, pain scores, etc.).

Unlike the previous version which mentioned only about Boolean and key terms, this version has more clarity on search in general. This change is again in sync with the principle of the update, the holistic approach.

\section{Discussion and Conclusion}

\section{How will this change work for the industry?}

Changes in the personnel and expertise requirements.

As per the new guidelines, clinical evaluation will be a multidisciplinary activity. There will be a need for medical device, technical and engineering expert, therapeutic area (medical) expert, regulatory expert, medical writer and literature search. The Table 2 below provides an outlook on how various skills will be used for various parts of the CER.

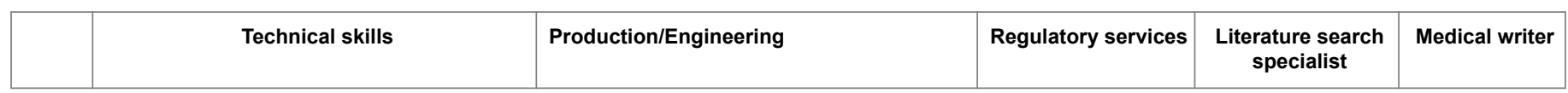


Citation: Ashish Indani (2017) European Council's Recent Revision in MedDev 2.7.1 Guidelines (Revision 4) - Quantifying its Change and its Impact. J Bioengineer \& Biomedical Sci 7: 225. doi:10.4172/2155-9538.1000225

Page 6 of 7

\begin{tabular}{|c|c|c|c|c|c|}
\hline & Basic understanding of clinical evaluation & & & & \\
\hline 1 & $\begin{array}{l}\text { Clinical evaluation, experience and evidence } \\
\text { and difference among them }\end{array}$ & Yes & Yes & No & Yes \\
\hline 2 & $\begin{array}{c}\text { Clinical research, phase and types of trials, } \\
\text { endpoints or outcomes, subsets }\end{array}$ & No & Yes & Yes & Yes \\
\hline 3 & $\begin{array}{l}\text { Biostatistics, published data, scopes and } \\
\text { limitations of published data and } \\
\text { comparability of datasets }\end{array}$ & No & No & Yes & Yes \\
\hline 4 & $\begin{array}{c}\text { Scientific terms and procedures for medical } \\
\text { devices evaluation at design and clinical } \\
\text { levels }\end{array}$ & Yes & No & No & Yes \\
\hline 5 & $\begin{array}{c}\text { Requirements SOPs, limitations, aims, } \\
\text { objectives and formats of clinical evaluation } \\
\text { report }\end{array}$ & No & Yes & No & Yes \\
\hline 6 & $\begin{array}{l}\text { Manufacturer's and user's perspective of } \\
\text { medical device performance and safety }\end{array}$ & No & Yes & No & Yes \\
\hline \multirow[t]{2}{*}{7} & $\begin{array}{c}\text { Design, technology and material information } \\
\text { of the device }\end{array}$ & Yes & No & No & No \\
\hline & Literature search, tabulation and appraisal & & & & \\
\hline 8 & $\begin{array}{c}\text { Set up Boolean logic with correct search } \\
\text { engines }\end{array}$ & No & No & Yes & No \\
\hline 9 & $\begin{array}{l}\text { Specificity or generality of a medical term to } \\
\text { be used as a key term }\end{array}$ & No & No & Yes & Yes \\
\hline 10 & $\begin{array}{c}\text { Complaints and events for medical devices } \\
\text { and various complaints databases for medical } \\
\text { devices }\end{array}$ & No & Yes & Yes & Yes \\
\hline 11 & $\begin{array}{c}\text { Ability to correctly broaden or narrow the } \\
\text { search or replacing key terms for better } \\
\text { outcomes }\end{array}$ & No & No & Yes & Yes \\
\hline 12 & $\begin{array}{c}\text { Distinguishing among various types of } \\
\text { publications such as article, review article, } \\
\text { abstract, presentation and their independence } \\
\text { value }\end{array}$ & No & No & Yes & Yes \\
\hline \multirow[t]{2}{*}{13} & $\begin{array}{l}\text { Various clinical trials registries and their use } \\
\text { for data mining }\end{array}$ & No & No & Yes & Yes \\
\hline & Clinical Evaluation & & & & \\
\hline 14 & $\begin{array}{c}\text { Therapeutic area and procedure to deploy the } \\
\text { device }\end{array}$ & No & No & No & Yes \\
\hline 15 & Establishing state-of-the-art & No & No & No & Yes \\
\hline 16 & $\begin{array}{l}\text { Performance, failure and safety incidences } \\
\text { linked with the device }\end{array}$ & No & Yes & No & Yes \\
\hline 17 & $\begin{array}{l}\text { Evaluate performance and safety of device } \\
\text { from reported clinical data sets }\end{array}$ & Yes & No & No & Yes \\
\hline 18 & $\begin{array}{l}\text { Establish substantial equivalence on basis of } \\
\text { clinical data }\end{array}$ & No & Yes & No & Yes \\
\hline \multirow[t]{2}{*}{19} & $\begin{array}{l}\text { Process of marketing authorization of devices } \\
\text { based upon equivalence }\end{array}$ & Yes & Yes & No & Yes \\
\hline & Writing Clinical Evaluation Report & & & & \\
\hline 20 & Regulatory guidelines & Yes & Yes & No & Yes \\
\hline
\end{tabular}




\begin{tabular}{|c|c|c|c|c|c|}
\hline 21 & Formatting and style guide & No & No & No & Yes \\
\hline 22 & $\begin{array}{l}\text { Experience in working on various clinical } \\
\text { regulatory documents and publications in the } \\
\text { past }\end{array}$ & No & No & No & Yes \\
\hline 23 & $\begin{array}{l}\text { Understanding of claim statements and } \\
\text { requirements to support claims }\end{array}$ & No & No & No & Yes \\
\hline 22 & $\begin{array}{l}\text { Understanding of end-to-end activities clinical } \\
\text { evaluation and clinical trials associated } \\
\text { Regulations and activities }\end{array}$ & No & Yes & No & Yes \\
\hline 24 & Citation and reference management & No & No & Yes & Yes \\
\hline \multirow[t]{3}{*}{25} & Project management & Yes & Yes & Yes & Yes \\
\hline & Total YES & 7 & 11 & 10 & 22 \\
\hline & Contribution from each function & $30 \%$ & $48 \%$ & $43 \%$ & $92 \%$ \\
\hline
\end{tabular}

Table 2: Task to skill mapping for CER writing process.

As mentioned in the guidelines, medical writers with 5-10 years' experience especially in writing should write CER. Now, every device, even if it does not require IFU, will need CER. Combination of these two amendments will increase the need of such personnel. However, the trends in personnel need can be considered in two phases: the immediate phase and th continuation phase. Many organizations develop CER for a few select devices. In the immediate phase, due to inclusion of all other devices, there will be a one-time increase in the number of CERs. In a continuation phase, there will be a need for update of CERs, frequency varying as per the device type. In this phase, there will be sporadic demand for the CER writers' skills. Therefore, unless there is multi-expertise, the organizations may opt to outsource the CER writing and updates activity.

Increased engagement: a need to shift to automation with the new guidelines, the manufacturer need to have increased engagement into the medical device reporting activity and materiovigilance. Most of the current pharmacovigilance platforms have a limited capacity to process medical device cases. Therefore, platforms developed for medical device vigilance or platforms customized to the need of manufacturer will be more popular for this service. In addition, automation of clinical evaluation report updates will be one of the areas to be looked in the near future, with some more efforts taken towards and uniform and integral medical device complaint management system (a clinical tool) and risk management platforms (a quality/ regulatory tool).

The updated guidelines have also included guidelines about postmarket activities. The manufacturer needs to engage more into PMS and PMCF. Current methods of conducting PMS and PMCF or device registries is very close to conduct of a phase IV study. Hence, their expenditure, process, approval and completion time etc. toll on time and finances. The manufacturers may look forward for tools developed to automate registries and PMS/PMCF programs and their product complaints capturing.
In the era of mobile phone applications, direct outreach to the end users and customers will help manufacturers to be in control of their product's safety and performance information in the real world.

\section{References}

1. (2016) MedDev 2.7.1 guidelines revision 4 European Commission.

2. (2010) MedDev 2.7.1 guidelines revision 3 from European Commission.

3. (2012) European Council Directive 90/385/EEC of 20 June 1990 relating to active implantable medical devices, Directive 93/42/EEC of 14 June 1993 concerning medical devices, Commission Regulation 722/2012 of 8 August 2012 concerning active implantable medical devices and medical devices manufactured utilizing tissues of animal origin 13: 301-310.

4. (2013) Commission Implementing Regulation 920/2013 of 24 September 2013 on the designation and the supervision of notified bodies under Council Directive 90/385/EEC on active implantable medical devices and Council Directive 93/42/EEC on medical devices PP: 8-19.

5. (2011) EN ISO 14155:2011 Clinical investigation of medical devices for human subjects - Good clinical Practice.

6. (2013) EN ISO 14971:2012 Medical devices - Application of risk management to medical devices.

7. (2013) MEDDEV 2.12/1 Guidelines on a medical devices vigilance system.

8. (2012) MEDDEV 2.12/2 Guidelines on post market clinical follow-up studies: A guide for manufacturer and notified body.

9. (2017) MEDDEV 2.7/2 Guidelines for competent authorities for making a validation/assessment of a clinical investigation application under directives 90/385/EEC and 93/42/EC.

10. (2007) GHTF SG5 N1R7:2007: Clinical evidence - Key definitions and concepts.

11. (2007) GHTF SG5 N2R8:2007: Clinical evaluation.

12. GHTF SG5 N41R9:2005: Essential principles of safety and performance. 